\title{
STEM Imaging of Magnetotactic Bacteria with the Fluid Cell
}

\author{
Sanjay Kashyap ${ }^{1}$, Teresa Perez-Gonzalez ${ }^{2}$, Damien Faivre, Lilah Rahn-Lee ${ }^{3}$, Arash Komeili , \\ and Tanya Prozorov
}

1. Emergent Atomic and Magnetic Structures, Division of Materials Sciences and Engineering, Ames Laboratory, Ames, IA 50011, USA

2. Department of Biomaterials, Max Planck Institute of Colloids and Interfaces, Science Park Golm, 14424 Potsdam, Germany.

3. Department of Plant and Microbial Biology, University of California, Berkeley, 111 Koshland Hall MC 3102, Berkeley, CA 94720, USA.

Magnetotactic bacteria, present in many natural aquatic environments, biomineralize ordered chains of uniform magnetite or greigite nanocrystals. Such nanoparticles exhibit nearly perfect crystal structures and consistent species-specific morphologies, leading to well-defined magnetic properties. As a result, magnetotactic bacteria can serve as a model system for the study of molecular mechanisms of magnetite biomineralization, with Transmission Electron Microscopy (TEM) providing vital information about the organization of the cellular structures. TEM traditionally does not allow imaging in native liquid or atmospheric environments because of the high vacuum requirements of the instrument. Even with advanced cryo-EM imaging, it is inherently difficult to distinguish the individual components in bacterial specimens due to notoriously low contrast of a biological matter.

We present, for the first time, imaging of live cells of magnetotactic bacteria in liquid using a fluid cell TEM holder platform (Hummingbird Scientific), with the bacterial cultures sealed between the two thin electron-transparent silicon nitride window membranes. High-Angle Annular Dark-Field Scanning Transmission Electron Microscopy (HAADF-STEM) and Bright Field Transmission Electron Microscopy are used to provide compositional and morphological information of the analyzed sample, as shown in Figure 1. Energy Dispersive X-ray Spectroscopy (EDS) and Energy Filtered Transmission Electron Microscopy (EF TEM) analysis and HAADF-STEM Electron Energy Loss Spectroscopy (EELS) were used for probing the localized chemical environment of the specimen ex-situ. The described approach is expected to have a direct impact on the understanding of biological processes by enabling in vivo characterization of a wide range of inorganic structures biomineralized by various microorganisms. 


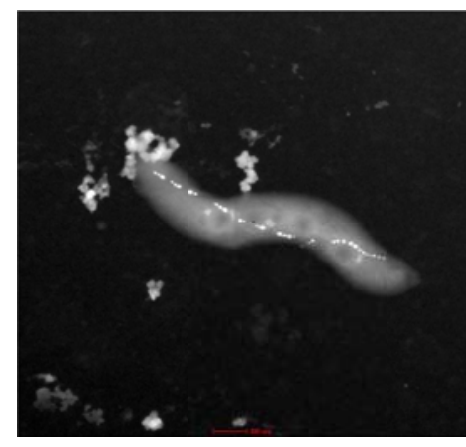

(a)

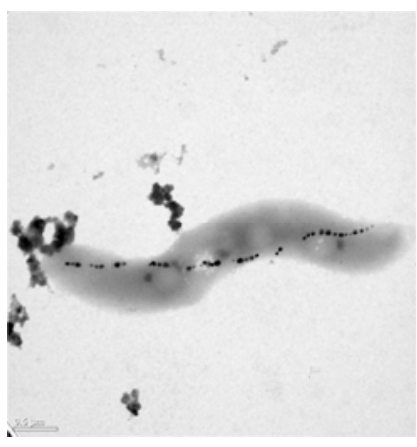

(b)

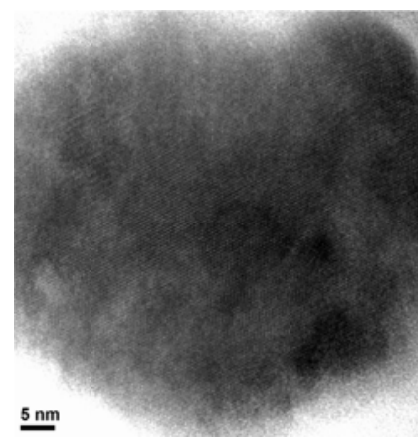

(c)

Figure 1. Magnetospirillum magneticum AMB-1 with the cell diameter of approximately $0.5 \mu \mathrm{m}$ and cell length of approximately $3 \mu \mathrm{m}$ in the fluid cell: (a) High-Angle Annular Dark-Field Scanning Transmission Electron Microscopic (HAADF-STEM) image of bacterial cell, (b) bright field Transmission Electron Micrograph of the same cell. An intracellular chain of over 15 magnetite nanocrystals (magnetosomes) with an average diameter of $50 \mathrm{~nm}$ can be clearly seen in both images; (c) bright field TEM image of the individual magnetosome magnetite nanocrystal.

1. The work in Ames Laboratory was supported by the U.S. Department of Energy, Basic Energy Sciences, Materials Sciences and Engineering Division. The Ames Laboratory is operated for the U.S. Department of Energy by Iowa State University under contract DEAC02-07CH11358. 